\title{
HÁBITO ALIMENTAR DO AGULHÃO-VERDE, Tetrapturus Pfluegeri (ROBINS \& DE SYLVA, 1963), (TELEOSTEI-ISTIOPHORIDAE), CAPTURADO NO ATLÂNTICO OESTE TROPICAL
}

Recebido em: julho/2013

Aceito em: agosto/2013

Fernanda Virginia Albuquerque SILVA ${ }^{1 *}$

Patrícia Barros PINHEIRO ${ }^{1}$

Natalia Priscila Alves BEZERRA ${ }^{1}$

Teodoro VASKE JUNIOR ${ }^{2}$

Antonio de Lemos Vasconcelo Filho ${ }^{3}$

Fábio Hissa HAZIN ${ }^{1}$

\begin{abstract}
RESUMO
O agulhão verde (Tetrapturus pfluegeri) representa uma das maiores capturas pela frota atuneira que opera com espinhel pelágico no Atlântico. Apesar de não ser alvo da pesca comercial de atuns, o agulhão verde é capturado como fauna acompanhante. Ao total, foram analisados de 182 estômagos de agulhões verde com amplitude de tamanho variando de 144 a $210 \mathrm{~cm}$ (MIF). Do total dos estômagos analisados $73,6 \%$ apresentaram algum conteúdo e $26,4 \%$ estavam vazios. Os peixes foram o grupo dominante, seguidos dos cefalópodes, com as presas variando de 3,4 a $50,1 \mathrm{~cm}$, a maioria das quais com comprimento entre 4,5 e 7,5 cm. Segundo O IIR o grupo

Teleostei foi o item mais importante, com $20,0 \%$ em número e ocorrendo em $41,0 \%$ dos estômagos seguidos da lula Hyaloteuthis pelagica, com $24,1 \%$ em número e $20,1 \%$ de ocorrência. O terceiro item em importância foram os exemplares da familia Scombridae, com $5,7 \%$ em número e ocorrendo em 13,4\% dos estômagos.De acordo com índice padronizado de Levins $(B=0,2)$ o agulhão verde pode ser caracterizado como um predador especialista. O T. pfluegeri mostrou ter um hábito alimentar predominantemente de Teleósteos e Cefalópodes, sendo os mais importantes o Katsuwonus pelamis e Hyaloteuthis pelagica.
\end{abstract}

Palavras chave: Hábito alimentar, estômagos, Istiophoridae

\section{ABSTRACT}

The longbill spearfish (Tetrapturus pfluegeri) represents one of the largest catches by tuna fleet operating in the Atlantic pelagic long line. Although not targeted on commercial fishing for tuna, the longbill spearfish are caught as bycatch. In total were analyzed 182 stomachs of individuals with amplitude in size varying from 144 to $210 \mathrm{~cm}$ low jaw fork length (LJFL). Of total stomachs examined, $73.6 \%$ had some content and $26.4 \%$ were empty. Fish were the dominant group, followed by cephalopods, with prey ranging from 3.4 to $50.1 \mathrm{~cm}$ and the most of with length between 4.5 and $7.5 \mathrm{~cm}$. According to Index of Relative
Importance (IRI), the Teleostei group was the most important item, with $20.0 \%$ in number and occurring in $41.0 \%$ of the stomachs, followed by squid Hyaloteuthis pelagica, with $24.1 \%$ in number and $20.1 \%$ of occurrence. The third item in importance were species of Scombridae Family, with $5.7 \%$ in number and occurred in $13.4 \%$ of stomachs. According to standardized Levins index $(B=0.2)$ the longbill spearfish can be characterized as a specialist predator. The $T$. pflueger ishowed a feeding habit predominantly of teleosts and cephalopods, the most important the Katsuwonu spelamis and Hyaloteuthis pelagica.

Keywords: Feeding habits, stomachs, Istiophoridae.

\footnotetext{
${ }^{1}$ Laboratório de Oceanografia Pesqueira, Universidade Federal Rural de Pernambuco. Rua Dom Manuel de Medeiros, s/n. Dois Irmãos, Recife, Pernambuco, Brasil, CEP: 52171-900;

fv.albuquerque85@gmail.com*,patriciabarros_pinheiro@hotmail.com, natalia_ufrpe@yahoo.com.br, fhvhazin@terra.com.br ${ }^{2}$ Laboratório de Elasmobrânquios e Nécton Marinho, Universidade Estadual Paulista - UNESP/CLP, Praça Infante Dom Henrique, s/n.Bitaru, São Vicente, São Paulo,Brasil, CEP: 1330-900; vaske@ig.com.br ${ }^{3}$ Departamento de Oceanografia, Universidade Federal de Pernambuco. Av. Arquitetura, S/n - Cidade Universitária Recife, Pernambuco, Brasil, CEP: 50740-550; antoniolvf@bol.com.br
} 
ALBUQUERQUE, F. V. et al. Hábito alimentar do agulhão-verde, Tetrapturus pfluegeri (Robins \& de Sylva, 1963), capturado no Atlântico Oeste Tropical

\section{INTRODUÇÃO}

O agulhão-verde, Tetrapturus pfluegeri, pertencente à Ordem Perciformes e à Família Istiophoridae (NAKAMURA, 1985), é uma espécie de reduzido tamanho, em relação aos demais membros da família, embora possa atingir comprimento total de $200 \mathrm{~cm}$ e pesar $45 \mathrm{~kg}$ (NAKAMURA, 1985). Amplamente distribuído no Oceano Atlântico, em águas tropicais e temperadas, apresenta uma amplitude latitudinal, inferida a partir das suas capturas por embarcações comerciais que operam com espinhel de superfície, que se estende aproximadamente entre $40^{\circ} \mathrm{N}$ e $35^{\circ} \mathrm{S}$ (NAKAMURA, 1985). É uma espécie oceânica, epipelágica e migradora, sendo geralmente encontrada em águas quentes acima da termoclina (entre 21 e $28^{\circ} \mathrm{C}$ ). Apesar de não ser alvo específico de nenhuma pescaria comercial, é capturado como fauna acompanhante, em função de se distribuir no mesmo habitat e apresentar dieta similar ao de outras espécies de elevado valor comercial, como o espadarte (Xiphias gladius) e os atuns (Thunnus spp.) (NAKAMURA, 1985; OVCHINNIKOV, 1971). Além das capturas realizadas pela pesca comercial atuneira, também é bastante capturado nas pescarias esportivas (NAKAMURA, 1985; De SYLVA, 1974), juntamente com os agulhões branco (Kajikia albida) e negro (Makaira nigricans).

Os estudos dos hábitos alimentares, por sua vez, são importantes para o entendimento da amplitude de uso de recursos ao longo da distribuição temporal e espacial de qualquer espécie (KREBS, 1989), e, consequentemente, da dinâmica das populações e comunidades (CUNNIGHAM, 1989), colaborando para o desenvolvimento de estratégias para o manejo sustentável dos ecossistemas marinhos (HAHN; DELARIVA, 2003).

O presente trabalho, portanto, teve como objetivo aprofundar os conhecimentos sobre o hábito alimentar do agulhão-verde capturado no Atlântico oeste tropical, com vistas a gerar subsídios capazes de contribuir não apenas com a conservação da espécie estudada, alvo de uma crescente mortalidade por pesca, mas para a compreensão da dinâmica trófica do ecossistema pelágico oceânico.

\section{MATERIAL E MÉTODOS}

As amostras foram obtidas no período de junho de 2008 a janeiro de 2012, por meio do Programa Nacional de Observadores de Bordo, do Ministério da Pesca e Aquicultura (MPA), com embarques efetuados nas embarcações estrangeiras que operam com espinhel pelágico, da frota atuneira brasileira, sediada no porto de Natal (RN).

Todos os indivíduos examinados foram sexados e mensurados quanto comprimento da mandíbula inferior até a forquilha (MIF) ainda a bordo das embarcações. Os estômagos foram coletados durante a evisceração dos exemplares e congelados para posterior análise em laboratório.

Já em laboratório, uma vez descongelados, os estômagos foram seccionados para a coleta do seu conteúdo. Após a identificação dos itens, o número de presas de cada item alimentar, assim como o comprimento do manto, para os cefalópodes $(\mathrm{cm})$, o comprimento total para outros organismos $(\mathrm{cm})$ e, sempre que possível, a biomassa de cada presa $(\mathrm{g})$ foram registrados. Com a progressão das coletas, uma curva de diversidade de presas foi elaborada para se observar o tamanho ótimo da amostra já havia sido obtido.

O grau de repleção estomacal foi estabelecido conforme a seguinte escala (VASKE, 2004):I- vazio;II- um quarto preenchido; III- dois quartos preenchidos; IV- três quartos preenchidos e V- cheio. A importância de cada item alimentar na dieta foi avaliada com base no Índice de Importância Relativa (IIR) (PINKAS et al., 1971) adaptado para peso (HYSLOP, 1980; HACUNDA, 1981), como segue:

$$
\mathrm{IIR}_{\mathrm{i}}=\% \mathrm{FO}_{\mathrm{i}} \times\left(\% \mathrm{~N}_{\mathrm{i}}+\% \mathrm{P}_{\mathrm{i}}\right)
$$

Onde $\% \mathrm{FO}_{i}$ é a porcentagem da frequência de ocorrência de cada item alimentar; $\% \mathrm{~N}_{i}$ é a porcentagem em número de presas ingeridas de cada item alimentar $\mathrm{e} \% \mathrm{P}_{\mathrm{i}}$ é a porcentagem em peso de presas ingeridas de cada item alimentar. 
ALBUQUERQUE, F. V. et al. Hábito alimentar do agulhão-verde, Tetrapturus pfluegeri (Robins \& de Sylva, 1963), capturado no Atlântico Oeste Tropical

Para o cálculo do IIR não foram considerados como conteúdo os bicos isolados e sem massa muscular dos cefalópodes, de forma a se evitar uma sobrestimativa em número deste grupo, devido a um eventual acúmulo de bicos oriundos de alimentação de horas ou mesmo de dias anteriores, decorrente do processo de digestão extremamente lento dos mesmos (VASKEJUNIOR; RINCÓN, 1998).

Com o objetivo de se identificar o nível relativo de especialização na dieta da espécie, a amplitude de nicho trófico (amplitude da dieta) foi estimada por meio do índice padronizado de Levins (KREBS, 1989), expresso por:

$$
B=\frac{1}{\sum_{\mathrm{i}=1}^{\mathrm{n}} \mathrm{Pij}^{2}}
$$

onde $B$ é a amplitude do nicho trófico; $P i=$ proporção do item alimentar $i ; n=$ número total de presas consumidas por determinado predador.Para uniformizar a medida do nicho trófico, aplicou-se a fórmula de Hurlbert (1978), como segue:

$$
B a=\frac{(B-1)}{(N-1)}
$$

onde $B a$ é a amplitude do nicho (padronizada); $N$ é o número total de recursos consumidos; e $B$ é a amplitude do nicho, que pode variar de 0 , quando uma espécie consumiu somente um tipo de presa, a 1, quando uma espécie consumiu de forma similar vários tipos de presas.

\section{RESULTADOS}

Foram analisados 182 estômagos de agulhões-verde capturados pela frota atuneira, com amplitude de tamanhos variando entre 114 a $210 \mathrm{~cm}$ (MIF) com moda na classe 165-170 (Fig. 1). Do total dos estômagos analisados, $73,6 \%$ (134) tiveram algum conteúdo e $26,4 \%$ (48) estavam vazios. Em relação ao grau de repleção estomacal, $41,9 \%$ (62) estavam com o grau II; $20,3 \%$ (30) com o grau de repleção III; 9,5\% (14) com grau IV; e 0,7\% (1) com grau V (Fig. 2).

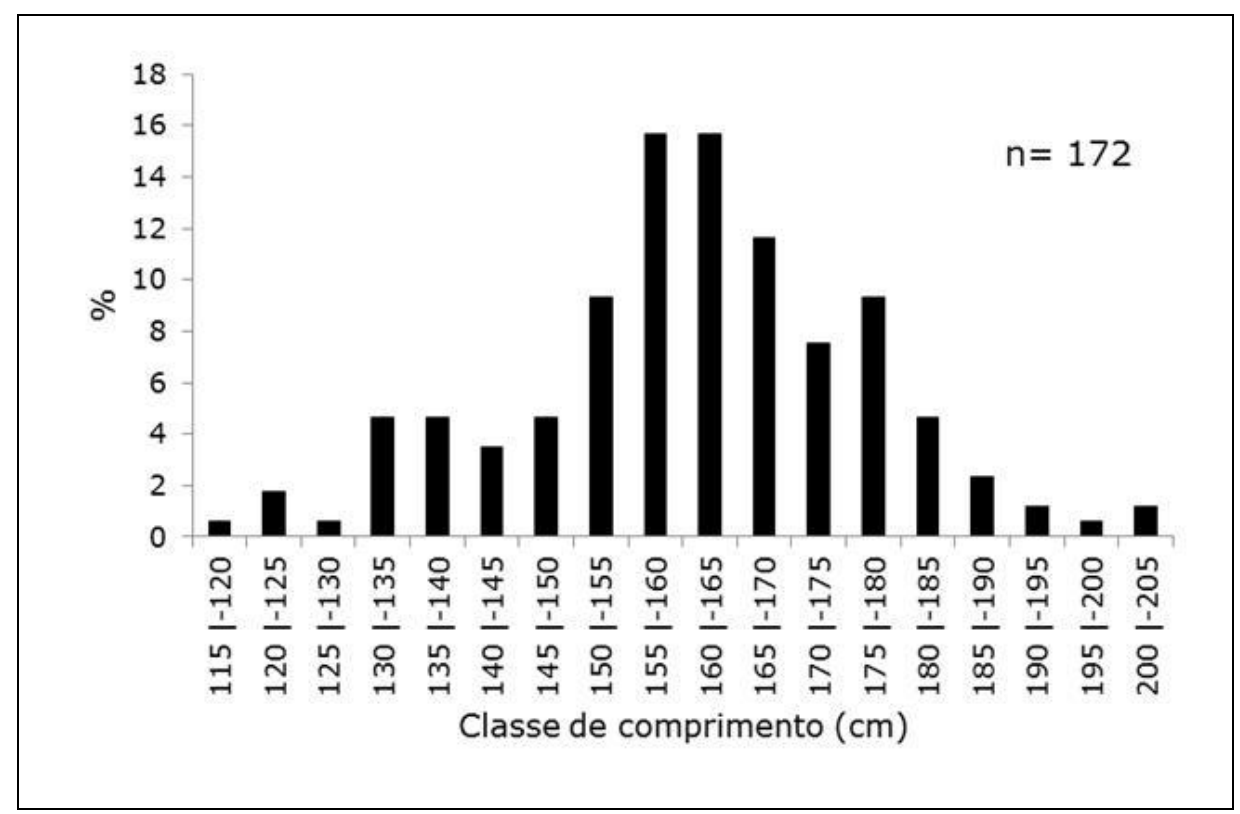

Figura 1- Distribuição de frequências de comprimento (MIF) do agulhão-verde capturado no Atlântico oeste tropical. 
ALBUQUERQUE, F. V. et al. Hábito alimentar do agulhão-verde, Tetrapturus pfluegeri (Robins \& de Sylva, 1963), capturado no Atlântico Oeste Tropical

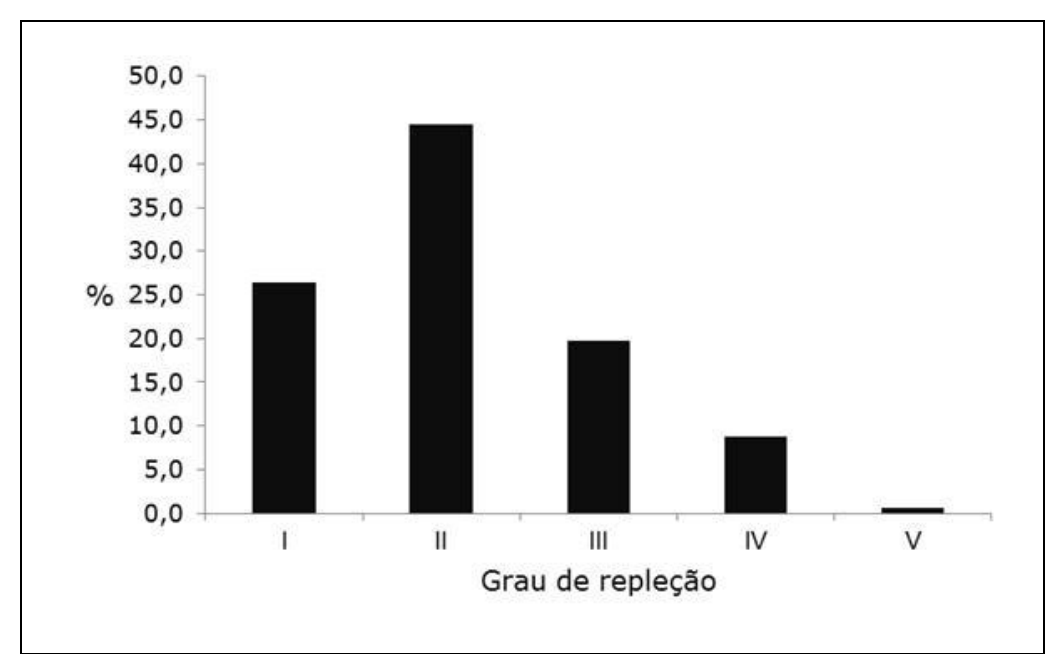

Figura 2 - Porcentagem de repleção estomacal do agulhão-verde capturado no Atlântico oeste tropical.

O agulhão-verde apresentou amplo espectro trófico, com 47 taxa (36 em nível de gênero ou espécie), sendo 20 de peixes, 15 de cefalópodes e 1 de crustáceo. Os grupos mais importantes foram os peixes, principalmente Katsuwonu spelamise Gempylus serpens, seguido dos cefalópodes, com destaque para a lula Hyaloteuthis pelagica.

Os peixes foram o grupo dominante em todos os indicadores (N, P, FO) representados na sua maioria por espécies epipelágicas. No cálculo do IIR (Tab. 1), Teleostei (indivíduos não identificados) foi o item mais importante, representando $20,0 \%$ em número e ocorrendo em $41,0 \%$ dos estômagos, seguidos dos exemplares da familia Scombridae, com 5,7\% em número e $13,4 \%$ de ocorrência. O terceiro item em importância entre os peixes foi Katsuwonu spelamis, com 3,9\% em número e 11,9\% em ocorrência. A ocorrência de peixes das Familias Myctophidae e Gonastomatidae indica que o $T$. pfluegeri também se alimenta de peixes mesopelágicos, sendo este item alimentar, porém, ocasional.

Os cefalópodes foram o segundo grupo em importância, segundo o IIR, representando $36,3 \%$ em número e $38,8 \%$ em frequência de ocorrência no total das presas. A família Ommastrephidae foi a mais representativa entre os cefalópodes, com destaque para Hyaloteuthis pelagica, que foi o principal representante, com $24,1 \%$ em número e ocorrendo em 20,1\% dos estômagos examinados, seguido dos polvos pelágicos representados por Ocythoe tuberculata com 7,0\% em número e 5,2\%, em ocorrência.

Os comprimentos das presas variaram entre 3,4 e 50,1 cm (Fig. 3), com uma maior frequência entre 4,5 e 7,5 cm, classes representadas principalmente por Dactylopterus volitans, Holocentrus adscensionis, Hyaloteuthis pelagica e Ocythoetu berculata (32,1\%). Os peixes da Família Scombridae e as espécies Gempylus serpens e Alepisau rusferox foram as presas com maiores comprimentos ( $>20,0 \mathrm{~cm}$ ).

O baixo valor no Índice padronizado de Levins $(B=0,2)$, calculado para a avaliação da amplitude da dieta, caracteriza o agulhão-verde como um predador especialista, com mais preferências pelo peixe bonito-listrado (Katsuwonus pelamis) e pela lula (Hyaloteuthis pelagica), embora tenha apresentado um amplo espectro de presas. A presença constante de estômagos com algum alimento reflete um hábito de se alimentar ativamente de presas isoladas e espaçadas no ambiente pelágico, uma vez que como predador pelágico ativo, gasta energia na natação e necessita estar sempre comendo para repor os gastos energéticos. 
ALBUQUERQUE, F. V. et al. Hábito alimentar do agulhão-verde, Tetrapturus pfluegeri (Robins \& de Sylva,

1963), capturado no Atlântico Oeste Tropical

Tabela 1- Itens alimentares do agulhão-verde com as respectivas porcentagens em número, peso e frequência de ocorrência de cada item $\left(\mathrm{N}^{\circ}=\right.$ número total, $\mathrm{P}=$ peso total, $\mathrm{FO}=$ frequência de ocorrência e $\mathrm{B}=\mathrm{Bico})$. Os dez itens mais importantes estão classificados de 1 a10. B indica apenas a ocorrência de bico. Epi=epipelágico; Meso=mesopelágico; Epimeso=epi emesopelágico.

\begin{tabular}{|c|c|c|c|c|c|c|c|c|}
\hline ITENS ALIMENTARES & Habitat & $\mathbf{N}^{\circ}$ & $\mathbf{N} \%$ & $\mathbf{P}$ & $\mathbf{P \%}$ & FO & FO\% & IRI \\
\hline Acanthuridae & Epi & 2 & 0,5 & 0,42 & 0,0 & 2 & 1,5 & \\
\hline \multicolumn{9}{|l|}{ Alepisauridae } \\
\hline Alepisauru sferox (Lowe, 1833) & Meso & 5 & 1,1 & 40,82 & 0,5 & 5 & 3,7 & \\
\hline Balistidae & Epi & 3 & 0,7 & 8,68 & 0,1 & 1 & 0,7 & \\
\hline Balistes capriscus (Gmelin, 1789) & Epi & 12 & 2,7 & 102,89 & 1,2 & 5 & 3,7 & 10 \\
\hline Balistes sp. & Epi & 1 & 0,2 & 0,45 & 0,0 & 1 & 0,7 & \\
\hline Belonidade & Epi & 1 & 0,2 & 20 & 0,2 & 1 & 0,7 & \\
\hline \multicolumn{9}{|l|}{ Bramidae } \\
\hline Brama brama (Bonnaterre, 1788) & Epimeso & 1 & 0,2 & 1,05 & 0,0 & 1 & 0,7 & \\
\hline Brama caribbea (Mead, 1972) & Epimeso & 18 & 4,1 & 100 & 1,2 & 12 & 9,0 & 6 \\
\hline Brama sp. & Epimeso & 11 & 2,5 & 30,91 & 0,4 & 1 & 0,7 & \\
\hline \multicolumn{9}{|l|}{ Carangidae } \\
\hline Decapterustabl (Berry, 1968) & Epi & 2 & 0,5 & 174,9 & 2,1 & 2 & 1,5 & \\
\hline Copleidae & Epi & 7 & 1,6 & 370,73 & 4,5 & 5 & 3,7 & 9 \\
\hline \multicolumn{9}{|l|}{ Coryphaenidae } \\
\hline Coryphaena hippurus (Linnaeus, 1758) & Epi & 2 & 0,5 & 115,15 & 1,4 & 2 & 1,5 & \\
\hline \multicolumn{9}{|l|}{ Dactylopteridae } \\
\hline Dactylopterus volitans (Linnaeus, 1758) & Epi & 8 & 1,8 & 8 & 0,1 & 7 & 5,2 & \\
\hline \multicolumn{9}{|l|}{ Diretmidae } \\
\hline Diretmu sargenteus(Johnson, 1864) & Meso & 1 & 0,2 & 3,53 & 0,0 & 1 & 0,7 & \\
\hline \multicolumn{9}{|l|}{ Diodontidae } \\
\hline Diodon spp. & Epi & 3 & 0,7 & 33,57 & 0,4 & 2 & 1,5 & \\
\hline Exocoetidae & Epi & 1 & 0,2 & 43,8 & 0,5 & 1 & 0,7 & \\
\hline Exocoetus volitans(Linnaeus, 1758) & Epi & 1 & 0,2 & 38,27 & 0,5 & 1 & 0,7 & \\
\hline Gempylidae & Meso & 6 & 1,4 & 32,08 & 0,4 & 2 & 1,5 & \\
\hline Gempylus serpens (Cuvier, 1829) & Meso & 36 & 8,2 & 256,85 & 3,1 & 13 & 9,7 & 5 \\
\hline Gonastomatidae & Meso & 2 & 0,5 & 2,78 & 0,0 & 2 & 1,5 & \\
\hline \multicolumn{9}{|l|}{ Hemiramphidae } \\
\hline Oxyporhamphus micropterus (Bruun, 1935) & Epi & 2 & 0,5 & 95,5 & 1,2 & 2 & 1,5 & \\
\hline \multicolumn{9}{|l|}{ Holocentridae } \\
\hline Holocentrus adscensionis(Osbeck, 1765) & Epi & 3 & 0,7 & 8,72 & 0,1 & 2 & 1,5 & \\
\hline Myctophidae & Meso & 8 & 1,8 & 13,57 & 0,2 & 4 & 3,0 & \\
\hline \multicolumn{9}{|l|}{ Molidae } \\
\hline Ranzania laevis(Pennant, 1776) & Epi & 10 & 2,3 & 497,09 & 6,0 & 6 & 4,5 & 8 \\
\hline Scombridae & Epi & 25 & 5,7 & 1615,81 & 19,5 & 18 & 13,4 & 3 \\
\hline Auxis sp. & Epi & 1 & 0,2 & 27,04 & 0,3 & 1 & 0,7 & \\
\hline Katsuwonus pelamis (Linnaeus, 1758) & Epi & 17 & 3,9 & 1976,94 & 23,9 & 16 & 11,9 & 4 \\
\hline Thunnus sp. & Epi & 2 & 0,5 & 449,64 & 5,4 & 2 & 1,5 & \\
\hline Teleostei & Epimeso & 88 & 20,0 & 1523,83 & 18,4 & 55 & 41,0 & 1 \\
\hline Peixes & & 279 & & 7593 & & & & \\
\hline
\end{tabular}


ALBUQUERQUE, F. V. et al. Hábito alimentar do agulhão-verde, Tetrapturus pfluegeri (Robins \& de Sylva, 1963), capturado no Atlântico Oeste Tropical

\begin{tabular}{|c|c|c|c|c|c|c|c|c|}
\hline $\begin{array}{l}\text { Brachyscelidae } \\
\text { Brachyscelus crusculum (Bate, 1861) }\end{array}$ & Epi & 1 & 0,2 & 0,04 & $>0,1$ & 1 & 0,7 & \\
\hline Crustáceos & & 1 & & 0,04 & & & & \\
\hline \multicolumn{9}{|l|}{ Argonautidae } \\
\hline Argonauta nodosa (Ligthtfoot, 1856) & Epi & 9 & 2,0 & 70,75 & 0,9 & 5 & 3,7 & \\
\hline Argonauta nodosa (B) (Ligthtfoot, 1856) & Epi & 10 & & & & 2 & & \\
\hline \multicolumn{9}{|l|}{ Bolitaenidae } \\
\hline Japetelladiaphana (B) (Hoyle, 1885) & Epimeso & 5 & & & & 3 & & \\
\hline \multicolumn{9}{|l|}{ Chiroteuthidae } \\
\hline Chiroteuthis sp. & Meso & 1 & 0,2 & 1,8 & 0,0 & 1 & 0,7 & \\
\hline Chiroteuthis sp. (B) & Meso & 1 & & & & 1 & & \\
\hline \multicolumn{9}{|l|}{ Cicloteuthidae } \\
\hline Discoteuthis discus (B) (Young \& Roper, 1969) & Meso & 1 & & & & 1 & & \\
\hline Cranchiidae & Meso & 2 & 0,5 & 17,93 & 0,2 & 2 & 1,5 & \\
\hline Taoniuspavo (B) (Lesuer, 1821) & Meso & 1 & & & & 1 & & \\
\hline \multicolumn{9}{|l|}{ Enoploteutidae } \\
\hline Enoploteuthis anapsis(B) (Roper, 1964) & Epimeso & 1 & & & & 1 & & \\
\hline Enoploteuthis leptura (Leach, 1817) & Epimeso & 2 & 0,5 & 9,72 & 0,1 & 1 & 0,7 & \\
\hline Enoploteuthis leptura (B) (Leach, 1817) & Epimeso & 1 & & & & 1 & & \\
\hline \multicolumn{9}{|l|}{ Histioteuthidae } \\
\hline Histioteuthis sp. & Epimeso & 1 & 0,2 & 1,8 & 0,0 & 1 & 0,7 & \\
\hline Histioteuthis sp. (B) & Epimeso & 7 & & & & 7 & & \\
\hline \multicolumn{9}{|l|}{ Ocythoidae } \\
\hline Ocythoe tuberculata (Rafinesque, 1814) & Epimeso & 31 & 7,0 & 114,57 & 1,4 & 7 & 5,2 & 7 \\
\hline Ocythoe tuberculata (B) (Rafinesque, 1814) & Epimeso & 33 & & & & 10 & & \\
\hline \multicolumn{9}{|l|}{ Ommastrephidae } \\
\hline Ommastrephidae (B) & Epimeso & 20 & & & & 2 & & \\
\hline Hyaloteuthis pelagica (Bosc, 1802) & Epimeso & 106 & 24,1 & 323,59 & 3,9 & 27 & 20,1 & 2 \\
\hline Hyaloteuthis pelagica (B) (Bosc, 1802) & Epimeso & 99 & & & & 28 & & \\
\hline Ommastrephes bartramii (Lesueur, 1821) & Epimeso & 1 & 0,2 & 7,71 & 0,1 & 1 & 0,7 & \\
\hline Ornithoteuthis antillarum(Adam, 1957) & Epimeso & 2 & 0,5 & 8,42 & 0,1 & 2 & 1,5 & \\
\hline Ornithoteuthis antillarum (B) (Adam, 1957) & Epimeso & 11 & & & & 2 & & \\
\hline Sthenoteuthis pteropus (Steenstrup, 1855) & Epimeso & 5 & 1,1 & 135,6 & 1,6 & 5 & 3,7 & \\
\hline Sthenoteuthis pteropus (B) (Steenstrup, 1855) & Epimeso & 1 & & & & 1 & & \\
\hline \multicolumn{9}{|l|}{ Tremoctopotidae } \\
\hline Tremoctopus violaceus (B) (Chiaie, 1830) & Epi & 6 & & & & 6 & & \\
\hline \multicolumn{9}{|l|}{ Vampyroteuthidae } \\
\hline Vampyroteuthis infernalis(B) (Chun, 1903) & Meso & 1 & & & & 1 & & \\
\hline Cefalópodes (com músculo) & & 160 & & 691,89 & & & & \\
\hline Cefalópodes (sem músculo) & & 198 & & & & & & \\
\hline TOTAL & & 638 & & 8284,9 & & & & \\
\hline
\end{tabular}


ALBUQUERQUE, F. V. et al. Hábito alimentar do agulhão-verde, Tetrapturus pfluegeri (Robins \& de Sylva, 1963), capturado no Atlântico Oeste Tropical

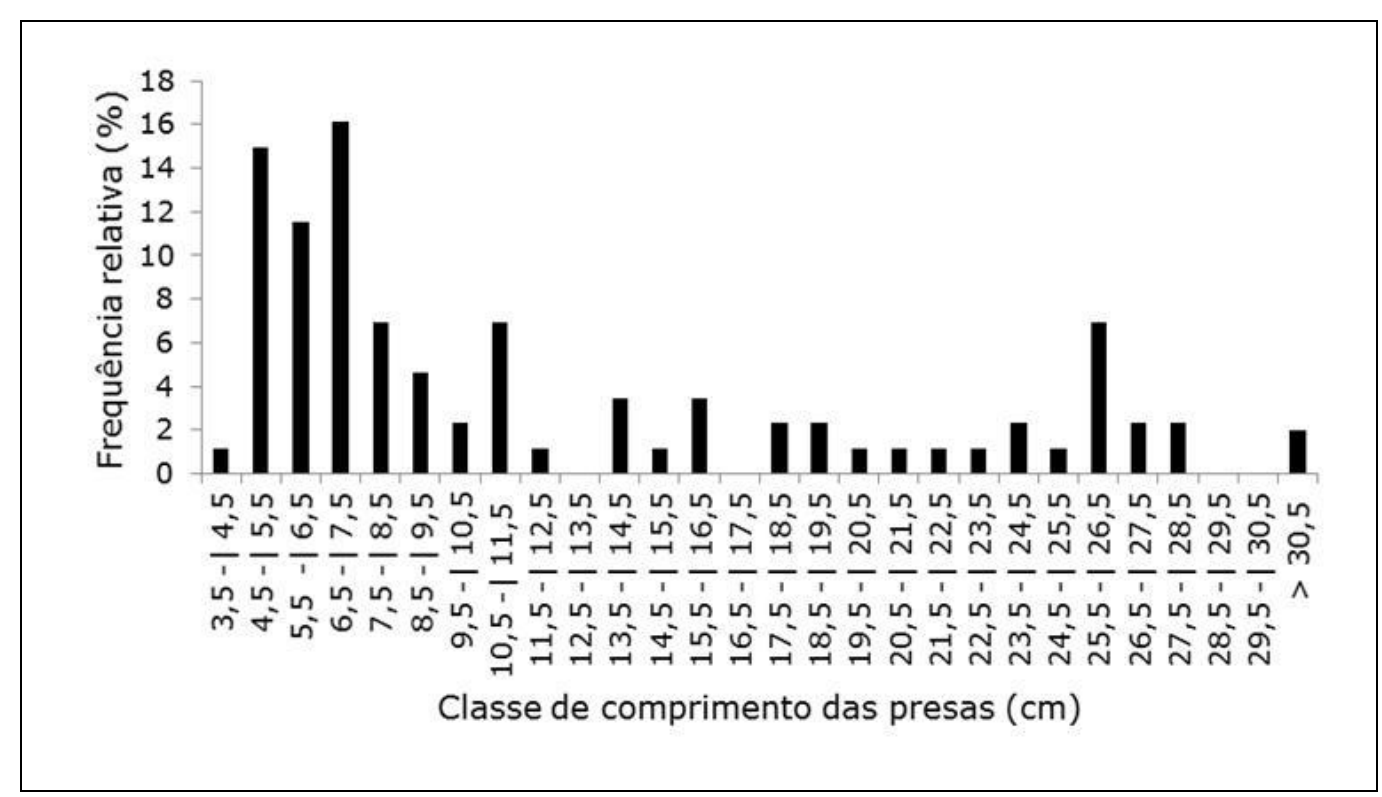

Figura 3 - Distribuição da frequência de comprimento das presas do agulhão-verde capturado no Atlântico oeste tropical.

\section{DISCUSSÃO}

Além da ampla distribuição horizontal no oceano Atlântico, o agulhão-verde faz ocasionalmente incursões em águas mais profundas, embora permaneça a maior parte do tempo na zona epipelágica (NAKAMURA, 1985). Segundo Lokkenborg e Byordal (1992), quanto maior o tamanho corporal do predador, maior o deslocamento e maior a área varrida na procura por alimento. Tal comportamento, típico de grandes predadores, como o agulhãoverde, aumenta a probabilidade de contato com artes passivas de pesca, como o espinhel e rede de emalhe. Esta capacidade de explorar amplas regiões em busca de alimento, inclusive em profundidades elevadas, é possível em função do grande tamanho dos olhos e da capacidade de conservação de calor corporal, permitindo que a espécie prede organismos que permanecem em camadas mais profundas, menos iluminadas e mais frias, onde outros predadores têm dificuldade de localização visual (CAREY, 1992). No presente estudo isto pôde ser constatado pela presença de cefalópodes mesopelágicos como os da família Cranchiidae, Chiroteuthi ssp., Histioteuthi ssp. e Japetelladia phananos estômagos. Entre os 14 taxa de cefalópodes encontrados, porém, quase todos fazem migração vertical permanecendo no epipelágico durante a noite e migrando à zona mesopelágica durante o dia com exceção dos polvos Tremoctopus violaceuse Ocythoe tuberculata que permanecem sempre no epipelágico (ROPER \& YOUNG, 1975). Com exceção dos peixes mesopelágicos Alepisaurus ferox, Diretmu sargenteus e os da família Gonastomatidae e Myctophidae, todos os outros peixes são representantes do ambiente epipelágico, de onde se pode concluir que o agulhão-verde procura se alimentar preferencialmente de peixes presentes nas camadas superficiais, realizando apenas ocasionalmente incursões em camadas mais profundas a procura de alimento, uma vez que cefalópodes e peixes mesopelágicos têm menor mobilidade, com estrutura corporal mais frágil e gelatinosa, oferecendoumaopção alimentar adicional, que exige menor gasto energético na captura.

Segundo Zavala-Camin (1981), os peixes representaram 67,1\% em número na dieta do agulhão-verde no sudeste e sul do Brasil. Posteriormente, Satoh et al. (2004) observaram $76,9 \%$ de participação de peixes no IIR da dieta do agulhão-verde no Atlântico Norte. Vaske et al. (2004), por sua vez, salientaram a importância dos peixes nos conteúdos estomacais de agulhão-verde no nordeste do Brasil por representarem 39,9\% em frequência numérica dos conteúdos, corroborando com os resultados encontrados no presente estudo.

A influência da proximidade dos bancos oceânicos, do Atol das Rocas e do Arquipélago de Fernando de Noronha nos espécimes amostrados foi a causa provável da ocorrência de organismos do micronécton na alimentação, particularmente dos peixes brefoepipelágicos 
ALBUQUERQUE, F. V. et al. Hábito alimentar do agulhão-verde, Tetrapturus pfluegeri (Robins \& de Sylva, 1963), capturado no Atlântico Oeste Tropical

representados por juvenis de Balistes capriscus, Dactylopterus volitans, Diodon sp. e Holocentrus adcencionis, uma vez que os adultos destas espécies habitamas águas mais costeiras. Bancos oceânicosfornecemzonas de abrigo, alimentação,e reprodução parao fitoplâncton,microzooplâncton, micronekton, peixes ecefalópodes (RODEN 1987, FONTENEAU, 1991,ROGERS, 1994). Esta concentração deorganismos em torno de ilhase bancos atrai atuns eoutros peixes pelágicos (HOLLAND et al.1999), entre os quais também o agulhão verde. No presente estudo, esta tendência foi evidenciada pela presença de peixes costeiros comuns em bancos e ilhas oceânicas encontrados nos estômagos dos espécimes examinados, aspecto facilitado também pela dispersão provavelmente ampla dasformas juvenis dessas espécies pelas correntes marinhas oceânicas. Tal fato indica que as formas jovens dessas espécies constituem parte importante da teia tróica pelágica, servindo como elo de transferências de energia do zooplancton e micronécton para os grandes predadores, como o agulhão-verde neste caso. Situação similar também foi observada em outros estudos de alimentação de outros predadores pelágicos na região (VASKE-JUNIOR e LESSA, 2004; VASKE-JUNIOR et al., 2004; PINHEIRO et al., 2010).

Como conclusão pode-se dizer que peixes e cefalópodes são os itens alimentares preferenciais do agulhão-verde no Oceano Atlântico oeste tropical, particularmente o tunídeo Katsuwonus pelamis e a lula Hyaloteuthis pelagica, podendo o mesmo, portanto, ser classificado como um carnívoro de primeira e segunda ordem.

\section{REFERÊNCIAS}

CAREY, F. G. Through the thermocline and back again. Heat regulation in big fish. Oceanus 35 (3): 79-85. 1992.

CUNNINGHAM, P. T. M. Observações sobre o espectro alimentar de Ctenosciaenagracilirrhus (Metzellar), Scianidae.Revista Brasileira de Biologia. 49 (2): 335-339. 1989.

De SYLVA, D. A review of the world sport fishery for billfishes (Istiophoridae and

Xiphiidae). In: R.S. Shomura and F. Williams (eds). Proc. Intl. Billfish Symp., Pt. 2. NOAA Tech. Rep. NMFS SSRF- 675, 335p. 1974.

FONTENEAU, A. Seamounts and tuna in the tropical eastern Atlantic. Aquat. Living Res. 4: 13-25. 1991.

HACUNDA. J. S. Trophic relationships among demersal fishes in a coastal area of the Gulf of Maine.Fishery Bulletin, 79, 775 - 788. 1981.

HAHN, N. S; DELARIVA, R. L. Métodos para avaliação da alimentação natural de peixes: O que estamos usando? Interciencia. 28 (2): 100-104p. 2003.

HOLLAND, K. N.; KLEIBER, P.; KAGIURA, S. M. Different residence times of yellowfin tuna, Thunnus albacares, and bigeye tuna, Thunnusobesus, found in mixed aggregations over aseamount. Fishery Bulletin. 97: 392-395. 1999.

HURLBERT, S. H. The measurement of niche overlap and some relatives. Ecology, 59(1): 67-77. 1978.

HYSLOP. E. J. Stomach content analysis - a review of methods and their application.Jornal of Fish Biology, 17: 411-429. 1980.

KREBS, C. J. Ecological Methodology. Harper e Row, New York. 1989.473p.

LOKKEBORG, S.; BYORDAL A. Species and size selectivity in longline fishing: a review. Fishery Research, 13:311-322. 1992.

NAKAMURA, I. FAO species catalogue. Vol. 5: Billfishes of the world. An annotated and illustrated catalogue of marlins, sailfishes, spearfishes and swordfishes known to date. FAO Fish. Synop., Rome, n.125, 65. 1985.

OVCHINNIKOV, V. V. Swordfishes and billfishes in the Atlantic Ocean. Ecology and Functional Morphology. Atlantic Scientific Research Institute of fisheries and Oceanography. 1971. 
PINHEIRO, P. B.; VASKE-JUNIOR, T.; HAZIN, F. H. V.; TRAVASSOS, P.; TOLOTTI, M. T.; BARBOSA, T. M. Diet of the white marlin (Tetrapturusalbidus) from the southwestern equatorial atlantic ocean.Collective Volume of Scientific Papers, ICCAT, Madrid, 65(5): 18431850. 2010.

PINKAS, L.; OLIPHANT, M. S.; IVERSON, I. L. K. Foodhabits of albacore, bluefin tuna anda bonito in California waters.Clif. Fish. Game, Fish. Bull., 152: 1971.105p.

RODEN, G. I. Effect of seamounts and seamount chains on ocean circulation and thermohaline structure. In: B.H. Keating, P. Fryer, R. Batiza\& G.W. Boehlert, (ed.) Seamounts, Islands and Atolls. Geophysical Monography 43, American Geophysical Union, Washington. p. 335-354. 1987.

ROGERS, A. D. The biology of seamounts. Adv. Marine Biology. v. 30: 306-3301994. ROPER, C. F. E.; YOUNG, R. E. Vertical distribution of pelagic cephalopods. Smithson. Contrib. Zool. 209: 1-51. 1975.

SATOH, K.; YOKAWA, K.; SAITO, H.; MATSUNAGA, H.; OKAMOTO, H.; UOZUMI, Y. Preliminary stomach contents analysis of pelagic fish collected by shoyo-maru 2002 research cruise in the atlantic ocean. Collective Volume of Scientific Papers, ICCAT, Madrid, 56(3): 1096-1114. 2004.

VASKE-JUNIOR, T.; RINCÓN-FILHO, G., Conteúdo estomacal dos tubarões azul (Prionace glauca) e anequim (Isurusoxyrinchus) em águas oceânicas do sul do Brasil.Revista Brasileira de Biologia. 58(3): 443-450. 1998.

VASKE-JUNIOR, T.; VOOREN, C. M.; LESSA, R. P. Feeding habits of four species of Istiophoridae (Pisces: Perciformes) from northeastern Brazil. Environmental Biology of Fishes 70, 293-304. 2004.

VASKE-JUNIOR, T.; LESSA, R. P. Feeding habits of the commomdolphinfish (Coryphaena hippurus), in the Northeastern Brazilian Exclusive Economic Zone. Arquivos de Ciências do Mar. 37: 131-138. 2004.

ZAVALA-CAMIN, L. A. Hábitos alimentares e distribuição dos atuns e afins (Osteichthyes - Teleostei) e suas relações ecológicas com outras espécies pelágicas das regiões sudeste e sul do Brasil. Tese de doutorado, Instituto de Biociências da Universidade de São Paulo, Brasil. 1981. 237 pp. 